\title{
EDITORIAL
}

\section{Welcome to the Annals of Family Medicine}

\author{
Kurt C. Stange, $M D, P b D,{ }^{1,2}$ William R. Pbillips, ${ }^{3,4}$ Louise S. Acheson, $M D, M S^{2,5}$ \\ Benjamin F. Crabtree, $P b D,{ }^{6}$ Stephen J. Zyzanski, $P b D,{ }^{2,7}$ William L. Miller, MD, MA, ${ }^{8}$ \\ Robin S. Gotler, $M A^{9}$ \\ ${ }^{1}$ Departments of Family Medicine, Epidemiology \& Biostatistics, and Sociology, Case Western Reserve University, Cleveland, Ohio \\ ${ }^{2}$ Ireland Comprehensive Cancer Center at University Hospitals of Cleveland and Case Western Reserve University, Cleveland, Ohio \\ ${ }^{3}$ Department of Family Medicine, University of Washington, Seattle, Wash \\ ${ }^{4}$ Centers for Public Health Evaluation and Research, Battelle Memorial Institute, Seattle, Wash \\ ${ }^{5}$ Departments of Family Medicine and Reproductive Biology, Case Western Reserve University, Cleveland, Ohio \\ ${ }^{6}$ Department of Family Medicine, University of Medicine \& Dentistry of New Jersey-Robert Wood Johnson Medical School, New Brunswick, NJ \\ ${ }^{7}$ Departments of Family Medicine, and Epidemiology \& Biostatistics, Case Western Reserve University, Cleveland, Ohio \\ ${ }^{8}$ Department of Family Medicine, Lehigh Valley Hospital, Allentown, Pa \\ ${ }^{9}$ Department of Family Medicine, Case Western Reserve University, Cleveland, Ohio
}

Ann Fam Med 2003;1:2-4. DOI: 10.1370/afm.46

\section{THE NEED FOR A GENERALIST FORUM}

\section{$\mathrm{T}$} The Annals of Family Medicine is a new peerreviewed research journal that aims to support a transdisciplinary learning community of those who generate and use new knowledge about health and primary health care. It is born out of the growing need to advance the knowledge base for the fundamental primary care functions and relationships. These relationships involve caring for individuals, families, and communities in ways that integrate, personalize, and prioritize health care to reduce the burden of illness. Family practice and the primary care disciplines need a forum to develop an integrated body of knowledge and a generalist para$\operatorname{digm}^{1}$ that is grounded in science, practice and different ways of knowing. ${ }^{2}$ The Annals aims to be such a forum.

The Annals features original research from the clinical, biomedical, social and health services sciences. Contributions on methodology and theory develop the underpinning for future research that advances the field. Selected systematic reviews draw on explicit methods to synthesize what is known and to move beyond - to advance theory or methods, or to identify new research directions. Essays from reflective clinicians, patients, families, communities, and policymak- ers portray a personal perspective on illness, health, and generalist health care.

\section{SUPPORTING AN INTERACTIVE LEARNING COMMUNITY}

The Annals features interactive online discussion groups called TRACK (Topical Response to the Annals Community of Knowledge). TRACK provides an opportunity for readers to actively engage in discussion of the implications of each published article. In addition to article-specific discussions, an open forum provides a mechanism for readers to introduce other important topics. This open forum also allows investigators to quickly publicize emerging findings and to gather input from those who are potentially affected by their work. Select aspects of the online discussions will be synthesized in subsequent issues of the Annals in a regular editorial feature called On TRACK. A limited number of submissions will be published as letters to the editor.

Both print and online versions of the new journal are being published 6 times a year, with plans to publish 10 issues per year in the future. The full-text online version is available free to all. This represents a unique opportunity for sharing new knowledge about health and primary care that is not limited by geo- 
graphical, financial or disciplinary boundaries. The print version is available by subscription and is free to members of the sponsoring organizations.

The Annals of Family Medicine Web site (http://www. annfammed.org) provides access to the online journal and the interactive discussion groups, as well as the peer reviewer registration form, information for authors, subscription information, and Rapid Review, our totally online manuscript submission-and-review process.

The Annals represents an unprecedented collaborative effort by 6 family medicine organizations coming together to support a forum for research and the intellectual development of the field. The Annals is overseen by an independent board of representatives from these 6 organizations: the American Academy of Family Physicians (AAFP), the American Board of Family Practice (ABFP), the Society of Teachers of Family Medicine (STFM), the Association of Departments of Family Medicine (ADFM), the Association of Family Practice Residency Directors (AFPRD), and the North American Primary Care Research Group (NAPCRG). These organizations share news and information in each issue.

The editorial and peer-review processes of the Annals have complete editorial independence from both organizational and commercial pressures. A distinguished Editorial Board helps to guide the journal's direction and foster transdisciplinary and international participation. A Readers and Contributors Advisory Group assists in keeping the Annals grounded in the needs of the diverse people and communities we serve.

The editors are grateful to the sponsoring organizations and their members for supporting this unique forum. We are indebted to the many authors who have shared their work with us and to the hundreds of peer reviewers who have provided the critical and constructive peer review that is the backbone of a scientific journal. We encourage others to sign up as peer reviewers and ask authors to continue to contribute their best work. We look forward to continuing to work with our experienced and collegial publishers at the American Academy of Family Physicians. Most of all, we look forward to publishing important research and reflections, and to supporting your online interactions. We appreciate the privilege of this stewardship role.

\section{IN THIS ISSUE}

Three articles in this issue relate to the important and quintessentially primary care problem of comorbidity. Starfield and colleagues, ${ }^{3}$ in a rigorous evaluation of health care for 11 important medical conditions, discovered that most health care visits are not for the selected condition, but are for other illnesses.
Moreover, the majority of visits for both the index condition and the comorbid illness are to primary care physicians. In a study of the patient perspective, conducted with rigorous qualitative research methods, Bayliss and collaborators ${ }^{4}$ identified important barriers to self-care among patients with multiple chronic illnesses. Many of these barriers are specifically related to having multiple medical conditions and may be worsened by health care systems that foster fragmentation rather than integration. The methodology paper by Rosen et $\mathrm{al}^{5}$ provides a theoretical framework for risk adjustment that is relevant to primary care. This framework is sensitive to the diverse clinical and nonclinical factors that affect health risk and the need for health care. Together, these articles identify the need for an approach to health care that is "oriented more toward patients' overall health care needs rather than diseases."3 Grumbach, in his insightful editorial, ${ }^{6}$ highlights the need for primary care practices and the larger health care system to develop approaches to integrating care for the multiple health problems faced by the people we serve. He challenges us to work together to develop systems to support care of whole people.

In other original research in this issue, 2 randomized controlled clinical trials call into question common clinical practices. Dickerson and colleagues found no difference in hyperglycemic or hypoglycemic events for hospitalized patients with type 2 diabetes using a sliding-scale insulin regimen and usual medications compared with those using routine diabetes medications alone. ${ }^{7}$ The findings build on similar research in patients with type 1 diabetes and empower clinicians and their patients to consider a more familiar and less expensive mode of care. In another trial, Volk and colleagues evaluated a decision aid for men making the complicated choices involved in prostate cancer screening. ${ }^{8}$ This patient-focused educational intervention increased knowledge about prostate cancer screening and reduced the rate of prostate-specific antigen testing. Apparently when men are more fully informed, they are less likely to desire testing.

The study by Leeman and Leeman shows the importance of the community context for primary care practice. ${ }^{9}$ The authors' elegant epidemiological study of a Native American community discovered that a low $(7 \%)$ rate of cesarean delivery is not due to a low prevalence of risk factors, but may be attributable to cultural and health care system factors, including, among others, nearly universal acceptance of trial of labor after cesarean

The essay by Scott and Crommet in this issue's "Reflections" relates the compelling story of how a patient's illness transformed both the patient and his family physician. ${ }^{10}$ The striking effect of the illness in 
redirecting Mr. Crommet's creativity from dancing to painting can be seen in his artwork, which is shown in color on the Annals website.

We look forward to working with you as readers, reviewers, authors and discussion participants. Please contribute your perspective at www.annfammed.org.

To read commentaries or to post a response to this article, see the online version at http://www.annfammed.org/cgi/content/full/1/1/2.

\section{References}

1. Kuhn TS. The Structure of Scientific Revolutions. Chicago, III: University of Chicago Press; 1966.

2. Stange KC, Miller WL, McWhinney I. Developing the knowledge base of family practice. Fam Med 2001;33:286-297.

3. Starfield B, Lemke KW, Bernhardt T, Foldes SS, Forrest CB, Weiner JP. Comorbidity: implications for the importance of primary care in "case" management. Ann Fam Med 2003;1:8-14.
4. Bayliss EA, Steiner JF, Fernald DH, Crane LA, Main DS. Descriptions of barriers to self-care by persons with comorbid chronic diseases. Ann Fam Med 2003;1:15-21.

5. Rosen AK, Ried R, Broemeling A-M, Rakovski CC. Applying a risk adjustment framework to primary care: can we improve on existing measures?. Ann Fam Med 2003;1:44-51.

6. Grumbach K. Chronic illness, Comorbidities, and the need for medical generalism. Ann Fam Med 2003;1:4-7.

7. Dickerson LM, Xiaobu Y, Sack JL, Hueston WJ. Glycemic control in medical inpatients with type 2 diabetes mellitus receiving sliding scale insulin regimens versus routine diabetes medications: a multicenter randomized controlled trial. Ann Fam Med 2003;1:29-35.

8. Volk RJ, Spann SJ, Cass AR, Hawley ST. Patient education for informed decision making about prostate cancer screening: a randomized controlled trial with one-year follow-up. Ann Fam Med $2003 ; 1: 22-28$

9. Leeman LM, Leeman R. A Native American community with a 7\% cesarean delivery rate: does case mix, ethnicity or labor management explain the low rate? Ann Fam Med 2003;1:36-43.

10. Scott JC, Crommet P. The face of cancer. Ann Fam Med 2003;1:52-54.

\title{
EDITORIAL
}

\section{Chronic Illness, Comorbidities, and the Need for Medical Generalism}

\author{
Kevin Grumbach, MD \\ University of California, San Francisco, School of Medicine, San Francisco, Calif \\ Conflicts of interest: None reported
}

Ann Fam Med 2003;1:4-7. DOI: 10.1370/afm.47

$\tau$ $t$ is said that when students enter medical school, they care about the whole person, and by the time they graduate, all they care about is the hole in the person. Current medical education inculcates many of the dominant values of modern medicine: reductionism, specialization, mechanistic models of disease, and faith in a definitive cure. As Fitzhugh Mullan observes, ${ }^{1}$ these values in medicine are part of a wider societal march toward reductionism and specialization. These trends are apparent in the fractioning of automotive repair shops into engine, transmission, and exhaust system specialists, and the need to find 3 different lawyers to prepare a will, settle a property dis-

\section{CORRESPONDING AUTHOR}

Kevin Grumbach, MD

San Francisco General Hospital, Ward 83

1001 Potrero Ave

San Francisco, CA 94110

keving@itsa.ucsf.edu pute, and incorporate a small business. Family medicine emphasizes a different world view, that of generalism. Mullan notes that "generalism as a phenomenon is not limited to medicine. To some extent, there is a competition in all human endeavor between the instinct to keep things whole, complete, and general, and the tendency to distinguish, sort, and reduce.

Generalism in human terms can be defined as a tendency to remain broadly focused, protean, and varied in world view and activity. The generalist is interested in the big picture with all of its nuances, connections, and complexities." 1 The whole, not just the holes.

Although a generalist perspective always has been important in health care, this broader view has become imperative in the face of the changing epidemiology of illness in industrial societies. Chronic conditions, not acute ailments, are now the most common problems in health care. The acute infection caused by a single microbe that can be definitively identified and eradi- 